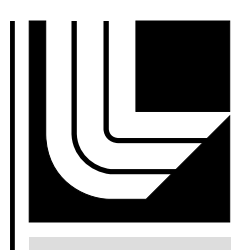

LAW RENCE LIVERMORE N A T IO N A L LABORATORY

\title{
Timing shifts due to NIF beam repointing
}

Jeffrey Koch

August 17, 2007 
This document was prepared as an account of work sponsored by an agency of the United States Government. Neither the United States Government nor the University of California nor any of their employees, makes any warranty, express or implied, or assumes any legal liability or responsibility for the accuracy, completeness, or usefulness of any information, apparatus, product, or process disclosed, or represents that its use would not infringe privately owned rights. Reference herein to any specific commercial product, process, or service by trade name, trademark, manufacturer, or otherwise, does not necessarily constitute or imply its endorsement, recommendation, or favoring by the United States Government or the University of California. The views and opinions of authors expressed herein do not necessarily state or reflect those of the United States Government or the University of California, and shall not be used for advertising or product endorsement purposes.

This work was performed under the auspices of the U.S. Department of Energy by University of California, Lawrence Livermore National Laboratory under Contract W-7405-Eng-48. 
To: $\quad$ Distribution

From: $\quad$ Jeffrey Koch

Subject: $\quad$ Timing shifts due to NIF beam repointing

Date: $\quad$ August 15, 2007

\section{Summary}

Repointing a NIF beam to hit a target position off target chamber center (TCC) will introduce a timing shift due to changes in the light pathlength. This shift could be important for target experiment requirements even for targets placed at TCC, since beam timing test shots will place beams up to $15 \mathrm{~mm}$ off TCC in order to spatially separate them on foil targets. In particular, timing errors due to beam repointing need to be considered against the $30 \mathrm{ps}$ RMS timing requirement. Since the repointing process will keep the beam passing through a fixed point in the final optics assembly (the conversion crystal) by tip/tilt adjustments of two turning mirrors (LM5 and LM7), the problem naturally divides into two parts: Timing offsets past the conversion crystal due to target positioning changes, and timing offsets behind the fixed point on the conversion crystal due to turning mirror adjustments. Timing offsets past the conversion crystal can be significant, but are trivial to calculate exactly; however, an exact calculation of timing offsets behind the fixed point on the conversion crystal would require a three-dimensional optomechanical raytrace model to be developed for every beamline, and this would be difficult and expensive. In this memo, I estimate the magnitude of timing offsets due to pathlength changes behind the conversion crystal by analysis of a worst-case model. I conclude that these timing offsets are insignificant compared with the current allocation in the $30 \mathrm{ps}$ RMS timing requirement, and that more detailed raytrace modeling of individual beams is not necessary.

I first work through an idealized model problem that can be solved analytically, and then work through a model problem more closely matching the conditions of the NIF beamlines. Figure 1 shows the idealized problem. The laser beam reflects off LM5 (top mirror), which is assumed to tilt about beam center, and then walks along the surface of LM7 (bottom mirror). LM7 tilts about the nominal beam center to repoint the beam to a fixed pivot point on the crystal. The angle $\phi$ is set by the desired lateral pointing offset about TCC. The presence of mirrors between LM5 and LM7, or after LM7, does not change the problem since they are fixed for all beam pointing positions. The mirrors do not in fact tilt about the beam centers, but I will explore the effects of that after solving this simpler problem first. 


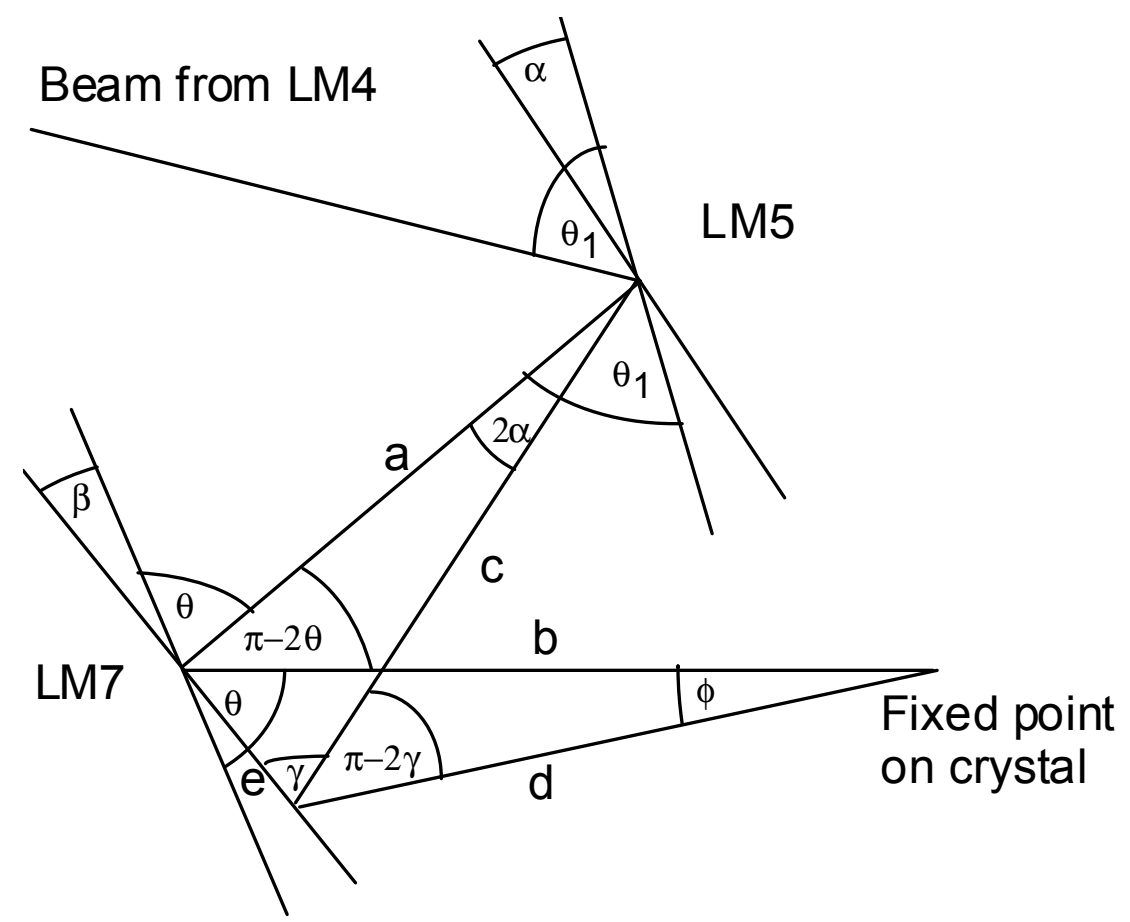

Figure 1: Idealized geometry for turning mirror offsets. Mirrors are assumed to pivot about the nominal beam center points.

Referring to the Fig. 1, I want to calculate the timing offset by calculating the pathlength difference $\Delta=(\mathrm{a}+\mathrm{b})-(\mathrm{c}+\mathrm{d})$, assuming $\mathrm{a}, \mathrm{b}, \theta_{1}, \theta$ and $\phi$ are known. Working through the angles to find $\gamma$ and $\beta$,

$$
\begin{aligned}
& 2 \alpha+\pi-2 \theta+\theta-\beta+\gamma=\pi \\
& \Rightarrow \gamma=\theta+\beta-2 \alpha \\
& \phi+\theta-\beta+\pi-\gamma=\pi \\
& \Rightarrow \gamma=\theta-\beta+\phi \\
& \Rightarrow \beta=\alpha+\phi / 2, \quad \gamma=\theta-\alpha+\phi / 2
\end{aligned}
$$

To find $\alpha$ and therefore $\gamma$ and $\beta$ in terms of known quantities, I can use the law of sines.

$$
\begin{aligned}
& \frac{\sin 2 \alpha}{e}=\frac{\sin \gamma}{a}, \frac{\sin \phi}{e}=\frac{\sin \gamma}{b} \\
& \Rightarrow \sin 2 \alpha=\frac{b}{a} \sin \phi
\end{aligned}
$$

To calculate $\mathrm{c}$ and $\mathrm{d}$, I can again use the law of sines. 


$$
\begin{aligned}
& \frac{\sin (\theta-\beta)}{d}=\frac{\sin \gamma}{b} \\
& \Rightarrow d=\frac{b \sin (\theta-\beta)}{\sin \gamma} \\
& \frac{\sin (\theta+\beta)}{c}=\frac{\sin \gamma}{a} \\
& \Rightarrow c=\frac{a \sin (\theta+\beta)}{\sin \gamma}
\end{aligned}
$$

Therefore,

$$
\Delta=a\left(\frac{\sin (\theta+\beta)}{\sin \gamma}-1\right)+b\left(\frac{\sin (\theta-\beta)}{\sin \gamma}-1\right)
$$

This is exact but cumbersome, so I use small-angle approximations to simplify it. $\phi$ is small, and therefore so are $\alpha$ and $\beta$, so I can expand the sines and cosines to second order in these quantities.

$$
\begin{aligned}
& \sin (\theta+\beta)=\sin \theta \cos \beta+\cos \theta \sin \beta \approx \sin \theta\left(1-\beta^{2} / 2\right)+\beta \cos \theta \\
& \sin (\theta-\beta)=\sin \theta \cos \beta-\cos \theta \sin \beta \approx \sin \theta\left(1-\beta^{2} / 2\right)-\beta \cos \theta \\
& \sin \gamma=\sin (\theta-(\alpha-\phi / 2))=\sin \theta \cos (\alpha-\phi / 2)-\cos \theta \sin (\alpha-\phi / 2) \\
& \quad \approx \sin \theta\left(1-(\alpha-\phi / 2)^{2} / 2\right)-(\alpha-\phi / 2) \cos \theta
\end{aligned}
$$

So,

$$
\Delta=\frac{1}{\sin \gamma}\left[\begin{array}{l}
a \sin \theta-a \frac{\beta^{2}}{2} \sin \theta+a \beta \cos \theta+b \sin \theta-b \frac{\beta^{2}}{2} \sin \theta b-b \beta \cos \theta \\
-a \sin \theta-b \sin \theta+a \frac{(\alpha-\phi / 2)^{2}}{2} \sin \theta+b \frac{(\alpha-\phi / 2)^{2}}{2} \sin \theta \\
+a(\alpha-\phi / 2) \cos \theta+b(\alpha-\phi / 2) \cos \theta
\end{array}\right]
$$

using $\alpha \sim b \phi / 2 a$ and $\beta=\alpha+\phi / 2$, this is:

$$
\Delta=\frac{1}{\sin \gamma}\left\lfloor\begin{array}{l}
(a-b)(\alpha+\phi / 2) \cos \theta+(a+b)(\alpha-\phi / 2) \cos \theta \\
-(a+b)(\alpha+\phi / 2)^{2} \sin \theta / 2+(a+b)(\alpha-\phi / 2)^{2} \sin \theta / 2
\end{array}\right\rfloor
$$

The first two terms cancel, and the second two terms add to $-2 \alpha \phi$, which is already second order (there is no first-order offset). Therefore, I just use $\sin \gamma=\sin \theta$ in the denominator, with the simple result:

$$
\Delta=\frac{-(a+b) b \phi^{2}}{2 a}
$$


Note that this is independent of the angle of incidence $\theta$, and scales as $b(a+b) / a$.

If I assume the fixed point is the focus lens rather than the crystal (the difference is negligible compared to the other distances involved), and if I assume a $50 \mathrm{~mm}$ lateral pointing offset at TCC $7700 \mathrm{~mm}$ away from the lens, then $\phi=0.3720505$ degrees. Looking over tables of NIF optics positions in global coordinates [1], I find that the maximum value of $b(a+b) / a$ is $27662.503 \mathrm{~mm}$, and occurs for beams Q31T_B4 and Q26B_B5. For most beams, this quantity is less than half this value, and for some it is less than a third of this value. Both worst-case beams have the same geometry, so:

$\mathrm{a}=\mathrm{LM} 7-\mathrm{LM} 5$ distance $=20950.47 \mathrm{~mm}$

$\mathrm{b}=(\mathrm{LM} 7-\mathrm{LM} 8)+($ Lens-LM8) distance $=15778.7738 \mathrm{~mm}$

$\theta($ on LM7 $)=40.880$ degrees

Using the exact first boxed equation, I calculate:

$$
\Delta=-0.5832024 \mathrm{~mm}
$$

Using the 2nd-order approximate boxed equation, I calculate:

$$
\Delta=-0.5832033 \mathrm{~mm}
$$

These results only differ in the 6th decimal place, suggesting the 2 nd-order approximation is excellent.

This pathlength difference corresponds to a worst-case $\sim 1.9 \mathrm{ps}$ timing change. The fact that this number is small is reasonable, since by changing the LM5/LM7 mirrors we are effectively mapping out a spherical-surface approximation to a curved reflecting mirror that focuses at a fixed point on the tripler. Since focusing optics work by matching all pathlength differences, we are really calculating the spherical aberration term to a very large $\mathrm{f} / \#$ imaging mirror. This would be expected to be small.

The next level of approximation is to explore additional time offsets due to the fact that LM5 and LM7 do not in fact tilt about the beam center point, but about points offset and behind the beam center points. The geometry is shown in Figure 2. The mirrors are assumed to pivot about points offset laterally and in depth from the beam center points, and for simplicity I assume the nominal angles of incidence are the same on both LM5 and LM7. Offsets and angles are positive as drawn, but can be positive or negative. 


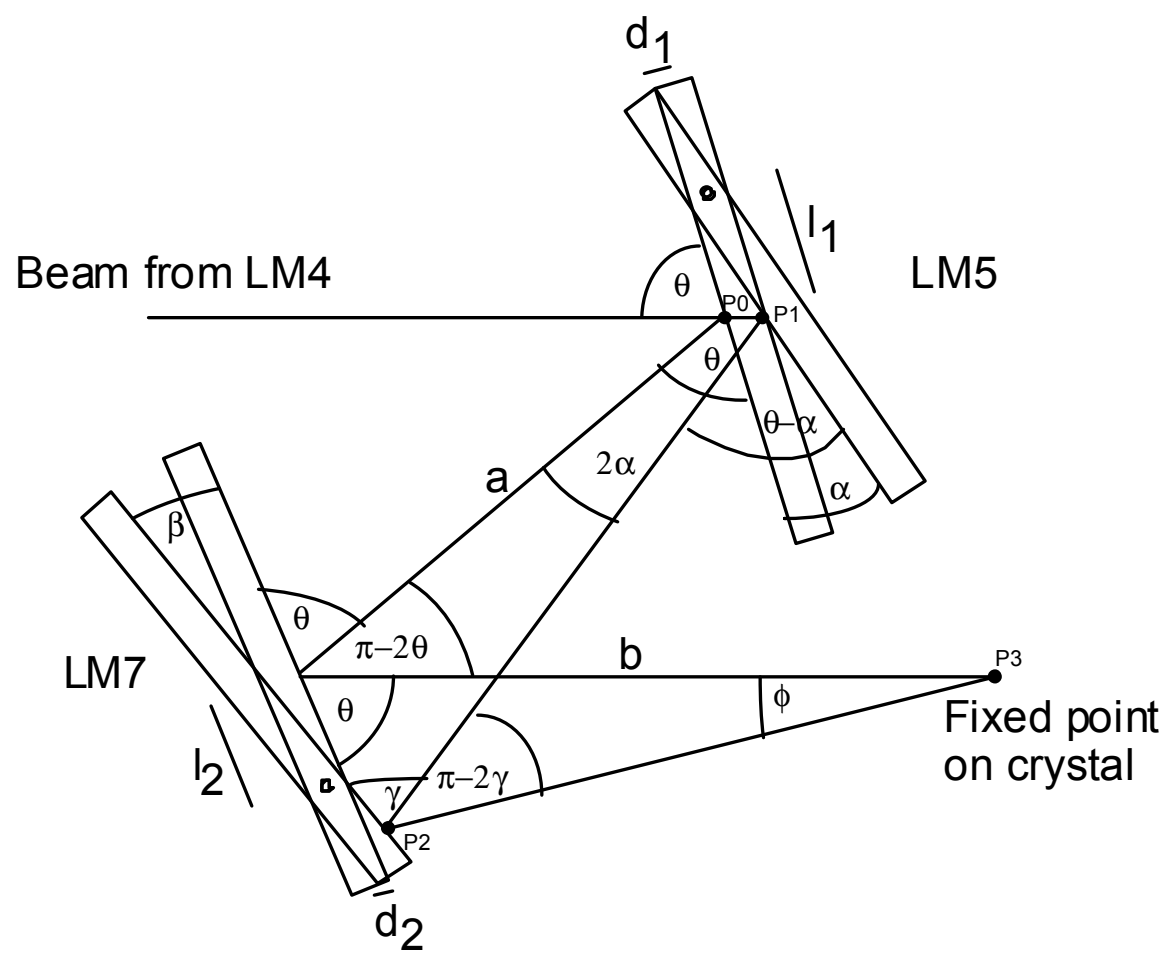

Figure 2: More realistic geometry for turning mirror offsets. Mirrors are assumed to pivot about points offset laterally and behind the nominal beam center points.

Due to the complexity of the math, I will approach this problem differently, and will calculate my final results with a computer program that iteratively solves for the time offsets. As before, working through the angles to find $\gamma$ and $\beta$,

$$
\begin{aligned}
& 2 \alpha+\pi-2 \theta+\theta-\beta+\gamma=\pi \\
& \Rightarrow \gamma=\theta+\beta-2 \alpha \\
& \phi+\theta-\beta+\pi-\gamma=\pi \\
& \Rightarrow \gamma=\theta-\beta+\phi \\
& \Rightarrow \beta=\alpha+\phi / 2, \quad \gamma=\theta-\alpha+\phi / 2
\end{aligned}
$$

Here, however, the geometry is too complicated to allow a simple relationship between $\alpha$ and $\phi$ to be derived. To solve this, I will instead use parametric equations for lines using fixed points $\mathrm{P} 0, \mathrm{P} 1, \mathrm{P} 2$, and $\mathrm{P} 3$ and corresponding ray vectors between the points that I can calculate directly. I solve for intersection points, sum up the total path between P0 and $\mathrm{P} 3$, and compare it to $(\mathrm{a}+\mathrm{b})$.

P0: I define this to be $(0,0)$, at the origin of coordinates. The incident vector is $<1,0>$. 
P1: The equation for the mirror surface, using the pivot point as a temporary origin, is:

$$
y^{\prime}=-x^{\prime} \tan (\theta-\alpha)-\frac{d_{1}}{\cos (\theta-\alpha)}
$$

The pivot point coordinates are transformed through:

$$
\begin{aligned}
& x^{\prime}=x+\left(l_{1} \cos \theta-d_{1} \sin \theta\right) \\
& y^{\prime}=y-\left(l_{1} \sin \theta+d_{1} \cos \theta\right)
\end{aligned}
$$

So the mirror surface equation is:

$$
y=-\left(x+\left(l_{1} \cos \theta-d_{1} \sin \theta\right)\right) \tan (\theta-\alpha)-\frac{d_{1}}{\cos (\theta-\alpha)}+\left(l_{1} \sin \theta+d_{1} \cos \theta\right)
$$

The intersection point $\mathrm{P} 1$ has $\mathrm{y}=0$, so

$$
\begin{gathered}
x 1=\frac{\left(l_{1} \sin \theta+d_{1} \cos \theta\right)}{\tan (\theta-\alpha)}-\left(l_{1} \cos \theta-d_{1} \sin \theta\right)-\frac{d_{1}}{\sin (\theta-\alpha)} \\
y 1=0
\end{gathered}
$$

The ray vector off LM5 is:

$$
r=\langle\cos 2(\theta-\alpha),-\sin 2(\theta-\alpha)\rangle
$$

P2: The equation for the mirror surface, using the pivot point as a temporary origin, is:

$$
y^{\prime \prime}=-x^{\prime \prime} \tan (\theta-\beta)+\frac{d_{2}}{\cos (\theta-\beta)}
$$

The pivot point coordinates are transformed through:

$$
\begin{aligned}
& x^{\prime \prime}=x^{\prime}-\left(l_{2} \cos \theta-d_{2} \sin \theta\right) \\
& y^{\prime \prime}=y^{\prime}+\left(l_{2} \sin \theta+d_{2} \cos \theta\right)
\end{aligned}
$$

So the mirror surface equation using it's own beam center point as the origin is:

$$
y^{\prime}=-\left(x^{\prime}-\left(l_{2} \cos \theta-d_{2} \sin \theta\right)\right) \tan (\theta-\beta)+\frac{d_{2}}{\cos (\theta-\beta)}-\left(l_{2} \sin \theta+d_{2} \cos \theta\right)
$$

The LM7 beam center coordinates are transformed through:

$$
\begin{aligned}
& x^{\prime}=x-a \cos 2 \theta \\
& y^{\prime}=y+a \sin 2 \theta
\end{aligned}
$$


So the mirror surface equation becomes:

$y=-\left(x-a \cos 2 \theta-\left(l_{2} \cos \theta-d_{2} \sin \theta\right)\right) \tan (\theta-\alpha-\phi / 2)+\frac{d_{2}}{\cos (\theta-\alpha-\phi / 2)}-\left(l_{2} \sin \theta+d_{2} \cos \theta\right)-a \sin 2 \theta$

The ray vector off $\mathrm{LM} 7$ is:

$$
r=\langle\cos \phi, \sin \phi\rangle
$$

To find P2, I create a parametric line from P1 and find the intersection point with this mirror plane. The parametric line is:

So the points are:

$$
\begin{aligned}
& x=x 1+t \cos 2(\theta-\alpha) \\
& y=-t \sin 2(\theta-\alpha)
\end{aligned}
$$

$$
\begin{aligned}
& x 2=x 1+\frac{\cos 2(\theta-\alpha)(C-x 1 \tan (\theta-\alpha-\phi / 2))}{\cos 2(\theta-\alpha) \tan (\theta-\alpha-\phi / 2)-\sin 2(\theta-\alpha)} \\
& y 2=\frac{-\sin 2(\theta-\alpha)(C-x 1 \tan (\theta-\alpha-\phi / 2))}{\cos 2(\theta-\alpha) \tan (\theta-\alpha-\phi / 2)-\sin 2(\theta-\alpha)}
\end{aligned}
$$

with

$$
C=\left(a \cos 2 \theta+\left(l_{2} \cos \theta-d_{2} \sin \theta\right)\right) \tan (\theta-\alpha-\phi / 2)+\frac{d_{2}}{\cos (\theta-\alpha-\phi / 2)}-\left(l_{2} \sin \theta+d_{2} \cos \theta\right)-a \sin 2 \theta
$$

P3: Here, I create a new parametric line from P2,

$$
\begin{aligned}
& x 3=x 2+t \cos \phi \\
& y 3=y 2+t \sin \phi
\end{aligned}
$$

I want to set $\mathrm{y} 3=-\operatorname{asin} 2 \theta$, so that fixes $\mathrm{t}$ and $\mathrm{x} 3$ :

$$
x 3=x 2-\frac{(a \sin 2 \theta+y 2)}{\tan \phi}
$$

Relative to the LM7 beam center point, the distance of this intersection point is

$$
\Delta x=x 2-\frac{(a \sin 2 \theta+y 2)}{\tan \phi}-a \cos 2 \theta
$$

If I've chosen the correct value of $\alpha$, then this will equal $b$. If I haven't, it'll be different from $b$, so I have to iterate. The next estimate of $\alpha$ is: 


$$
\alpha \rightarrow \alpha\left(1-\frac{(\Delta x-b)}{b}\right)
$$

I will stop iterating when

$$
\frac{(\Delta x-b)}{b}<1 e-7
$$

This will be accurate to a micron or so, which is more than sufficient.

\section{$\underline{\text { Results: }}$}

I coded these equations up in FORTRAN (see Appendix 1), using both positive and negative values for $11=243 \mathrm{~mm}$ (worst of two cases, the other is $187 \mathrm{~mm}$ ) and $12=138$ $\mathrm{mm}$ [2]. I also assume $\mathrm{d} 1=\mathrm{d} 2=40 \mathrm{~mm}$ [2]. I chose the same worst-case values of $\mathrm{a}, \mathrm{b}$ and $\theta$ that I used above, and assumed the fixed point is on the lens $7700 \mathrm{~mm}$ from TCC. To check the effect of the pivot depth, I also calculated the positive offset case with $\mathrm{d} 1=\mathrm{d} 2=0$. Finally, as a check, I also calculated the result for the idealized problem I worked out analytically above (no pivot point offsets), and the result agrees with my calculation. The curves are plotted in Figure 3.

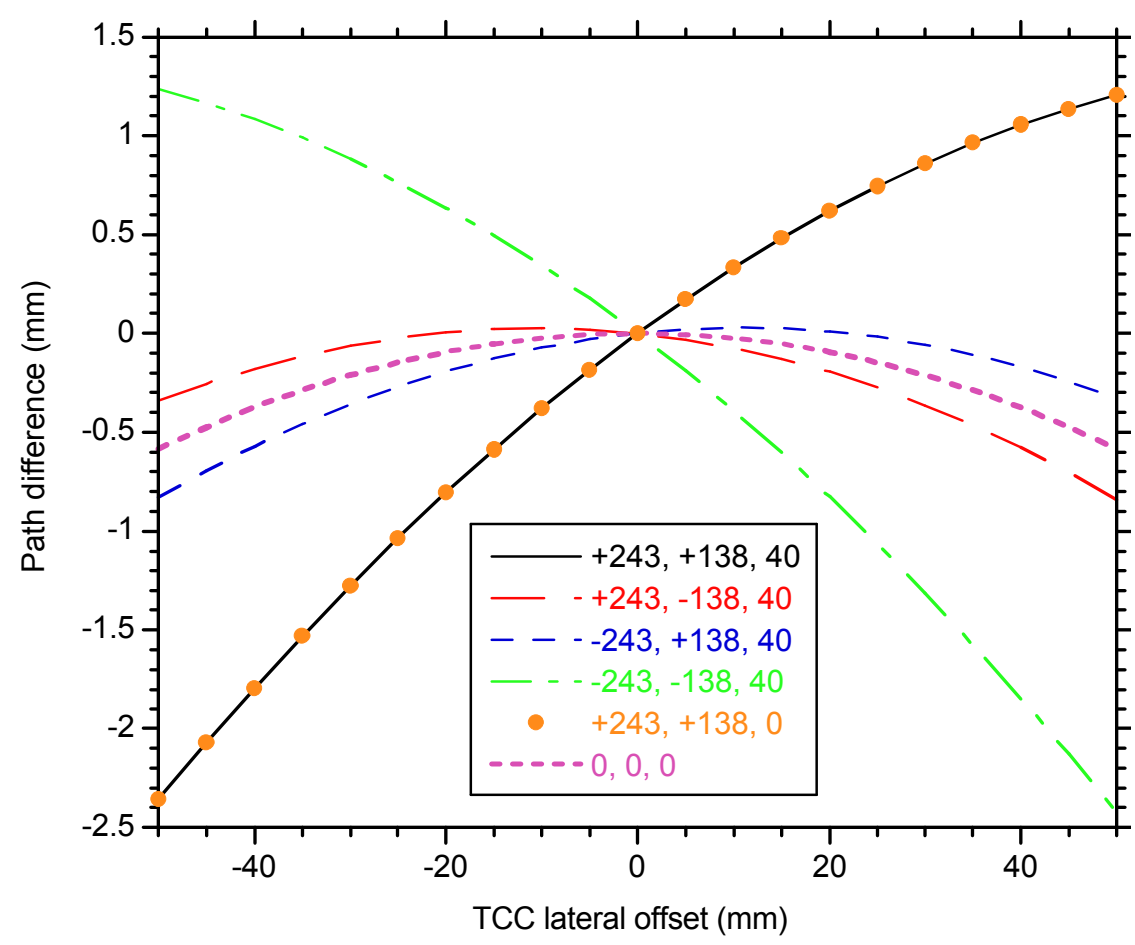

Figure 3: Path length difference versus lateral target position offset for several cases of pivot point offsets. 
I find that adding the effect of the pivot offsets can introduce an additional time offset in some cases, but the worst cases result in $\sim 2.4 \mathrm{~mm}$ pathlength differences, corresponding to $\sim 10$ ps timing offsets for $50 \mathrm{~mm}$ TCC target offsets.

The main concern for beam offset timing corrections is for beams pointed up to $15 \mathrm{~mm}$ laterally off TCC during timing shots to verify the $30 \mathrm{ps}$ RMS beam timing requirement for targets at TCC. The calculation above shows that for the very worst cases (worst choice of beams, maximum mirror pivot offsets, pivot offsets in directions that add the most pathlength difference, beams $+/-15 \mathrm{~mm}$ off TCC), the maximum timing offsets behind the conversion crystals are $3.5 \mathrm{ps} \mathrm{P}-\mathrm{V}$. The current allocation for this offset in the RMS spec is 3 ps [3], and therefore it appears that we do not need to calculate these offsets more precisely. The dominant contribution to timing offsets for targets placed away from TCC is therefore just the time-of-flight difference along the beam direction, and this can be calculated easily.

\section{References}

[1] NIF document NIF_5000875_BM_CEN_OE(1).xls, provided by John Villanueva. [2] Drawings provided by Erlan Bliss.

[3] Joe Holder, personal communication. 


\title{
Appendix 1: FORTRAN code.
}

\author{
program mirror
}

implicit none

double precision $\mathrm{p} i, \mathrm{x} l 1, \mathrm{x} 12, \mathrm{~d} 1, \mathrm{~d} 2, \mathrm{a}, \mathrm{b}, \mathrm{t}, \mathrm{x}, \mathrm{p}, \mathrm{al}, \mathrm{path}, \mathrm{x} 0, \mathrm{y} 0, \mathrm{x} 1, \mathrm{y} 1$,

$>$ t2, term, al2, $\mathrm{x} 2, \mathrm{y} 2, \mathrm{x} 3, \mathrm{y} 3$, dist, frac

integer $i$, istep

$\mathrm{pi}=\operatorname{dacos}(-1.0 \mathrm{~d} 0)$

c input variables

write $(6, *)$ 'enter pivot offset on LM5'

$\operatorname{read}\left(5,{ }^{*}\right) \times 11$

write $(6, *)$ 'enter pivot offset on LM7'

$\operatorname{read}(5, *) \times 12$

write $(6, *)$ 'enter pivot depth'

$\operatorname{read}(5, *) \mathrm{d} 1$

$\mathrm{d} 2=\mathrm{d} 1 \quad$ !assume depths are the same

$\mathrm{a}=20950.47 \mathrm{d0} \quad$ !distance between LM5 and 7

$\mathrm{b}=15778.7738 \mathrm{~d} 0$ !distance between LM7 and lens

$t=40.88 \mathrm{~d} 0$ !angle of incidence on LM7, assumed equal on LM5

$t=t \star p i / 180.0 \mathrm{~d} 0$

open (unit $=10$, file='pathdifference.txt', status='new')

C loop through $-50 \mathrm{~mm}$ to $50 \mathrm{~mm}$ in $5 \mathrm{~mm}$ TCC offset steps

do 50 istep $=-50,50,5$

$\mathrm{x}=\mathrm{dfloat}($ istep)

$\mathrm{p}=\mathrm{x} / 7700.0 \mathrm{~d} 0$ !angular offset in radians

make an initial guess for alpha

$\mathrm{al}=0.5 \mathrm{~d} 0 * \mathrm{~b} * \mathrm{p} / \mathrm{a}$

c loop until alpha converges on correct value to pivot about lens with angle phi do $100 \quad i=1,100$

path $=0.0 \mathrm{~d} 0$

$\mathrm{x} 0=0.0 \mathrm{~d} 0$

$\mathrm{y} 0=0.0 \mathrm{~d} 0$

$x 1=(x 11 * d \sin (t)+d 1 * d \cos (t)) / d \tan (t-a l)-x 11 * d \cos (t)+d 1 * d \sin (t)-d 1 / \sin (t-a l)$

$\mathrm{y} 1=0.0 \mathrm{~d} 0$

path $=$ path $+\mathrm{x} 1$

t2 $=2.0 \mathrm{~d} 0 * t$

term $=(a * d \cos (t 2)+x 12 * d \cos (t)-d 2 * d \sin (t)) * d \tan (t-a l-p / 2.0 d 0)+$

$>\quad d 2 / d \cos (t-a l-p / 2.0 d 0)-x l 2 * d \sin (t)-d 2 * d \cos (t)-a^{*} d \sin (t 2)$ $\mathrm{al} 2=2.0 \mathrm{~d} 0 * \mathrm{al}$

$\mathrm{x} 2=\mathrm{x} 1+\mathrm{dcos}(\mathrm{t} 2-\mathrm{al} 2) *(\operatorname{term}-\mathrm{x} 1 * \mathrm{dtan}(\mathrm{t}-\mathrm{al}-\mathrm{p} / 2.0 \mathrm{~d} 0)) /$

$>\quad(\operatorname{dcos}(t 2-a l 2) * d \tan (t-a l-p / 2.0 d 0)-d \sin (t 2-a l 2))$

y2 $=-d \sin (t 2-a l 2) *(\operatorname{term}-\mathrm{x} 1 * d \tan (t-a l-p / 2.0 \mathrm{~d} 0)) /$

$(\mathrm{dcos}(\mathrm{t} 2-\mathrm{al} 2) * \mathrm{dtan}(\mathrm{t}-\mathrm{al}-\mathrm{p} / 2.0 \mathrm{~d} 0)-\mathrm{dsin}(\mathrm{t} 2-\mathrm{al} 2))$

path $=$ path $+\operatorname{dsqrt}((x 2-x 1) * * 2+(y 2-y 1) * * 2)$

$x 3=x 2-(a * d \sin (t 2)+y 2) / d \tan (p)$

y3 $=-a * d \sin (t 2)$

path $=$ path $+\operatorname{dsqrt}((x 3-x 2) * \star 2+(y 3-y 2) * \star 2)$

dist $=x 3-a * d \cos (t 2)$

frac $=($ dist $-b) / b$

if (dabs (frac).1t.1.0d-7) then

goto 101

else

$a l=a l-f r a c * a l$

endif

continue

continue

write $(10, *) x$, path-a-b

50 continue

close (unit=10)

stop

end 